\title{
A CONSIDERATION ON THE FACTORS INFLUENCING THE PLAQUE SIZE IN A BACTERIOPHAGE (MT-BACTERIOPHAGE)
}

Tomie NOJIMA, SANETOShI MINAMIMOTO AND HIDEo FUKUMI

National Institute of Health, Tokyo,

(Received: January 18th, 1955)

INTRODUCTION

In a previous publication(1), we introduced an example in which the plaque size in a bacteriophage infection was influenced by the latent period in a given host-virus system. In this instance, the host microorganism splits off a kind of mutants at a certain mutation rate, on which the bacteriophage, named as $\boldsymbol{\pi}^{(\mathbf{2})}$ produces much smaller plaques than on the original culture, and corresponding with this, the latent period of the bacteriophage in its infection of the mutant cells was found to be longer than in the infection of the original culture. How ever, caution was also called in that paper to the fact that there were cases in which the difference of their latent periods does not necessarily reflect on their plaque size.

During the progress of the study, it has been recognized that the bacteriophage MT, isolated from a hen's stool through the infection of S. typhi (783), develops plaques whose size is different as they are produced either on $S$. typhi (783) or on S. pullorum (Nakamura). In the present paper, some consideration will be made on the factors affecting the plaque size in this bacteriophage MT infecting the Salmonellas mentioned above.

\section{EXPERIMENTAL MATERIALS AND METHODS}

The bacteriophage $M T$ : Isolated from a hen's stool by the usual way, using S. typhi (783) as a host.

The host microorganisms:

Salmonella typhi 783, O-form.

Salmonella pullorum (Nakamura).

Adams' description( ${ }^{(3)}$ was followed for conducting adsorption experiments, obtaining one-step-growth curves and other experiments of bacteriophages.

\section{EXPERIMENTAL RESULTS}

The plaque sizes of the bacteriophage MT on S. typhi (783) and on S. pullorum (Nakamura):

The plaques of the bacteriophage MT produced either on S. typhi (783) or S. pullorum (Nakamura) are plainly round with no halo, as shown in Fig. 1(a) and (b). The plaques on S. pullorum (Nakamura) are shown to be remarkably larger in diameter than those on $S$. typhi (783). The present paper is to discuss 
and consider what factor or factors are concerned with this plaque size difference.

The plating efficiency of the bacteriophage MT on S. typhi (783) and on S. pullorum (Nakamura):

The plating efficiency of the bacteriophage MT on $S$. typhi (783) is a little less than that on $S$. pullorum (Nakamura). This is evidenced by comparing the titration values of any sample of the MT phage both on $S$. typhi (783) and S. pullorum (Nakamura). Some examples are shown in Table 1.

Table 1. Plating efficiency of the phage MT to S. pullorum (Nakamura) and S. typhi (783)

\begin{tabular}{c|c|c}
\hline \multirow{2}{*}{$\begin{array}{c}\text { Preparation No. } \\
\text { of the phage }\end{array}$} & \multicolumn{2}{|c}{ Plaque count of the phage MT on } \\
\cline { 2 - 3 } & S. typhi (783) & S. pullorum (Nakamura) \\
\hline 1. & $2.5 \times 10^{8}$ & $3.8 \times 10^{8}$ \\
2. & $5.8 \times 10^{8}$ & $1.1 \times 10^{9}$ \\
3. & $3.2 \times 10^{8}$ & $4.1 \times 10^{9}$ \\
4. & $1.5 \times 10^{8}$ & $2.7 \times 10^{9}$ \\
5. & $1.0 \times 10^{8}$ & $5.0 \times 10^{7}$ \\
6. & $2.3 \times 10^{8}$ & $3.8 \times 10^{8}$
\end{tabular}

The adsorption of the bacteriophage MT to S. typhi (783) and to S. pullorum (Nakamura) :

It was already stated by us ${ }^{(4)}$ that the plating efficiency of the bacteriophage is an expression of the adsorption rate between phage particles and their host cells. The adsorption rates of the bacteriophage MT to S. typhi (783) and to S. pullorum (Nakamura) are shown in Fig. 2 as adsorption-time lines. They are considerably low in comparison with those of the usual bacteriophage, for instance, compared with the phage $\mathrm{T}_{1}{ }^{(5)}$. But, at any rate, the difference between them may seem to correspond with that of the efficiencies of plating of this bacteriophage on both bosts.

The latent periods and the burst sizes of the bacteriophage MT on the host cells S. typhi (783) and S. pullorum (Nakamura):

The one-step growth curves of the bacteriophage MT with its hosts $S$. typhi (783) and S. pullorum (Nakamura) were obtained by the usual procedure ${ }^{(3)}$, as shown in Fig. 3. As seen clearly in this figure, this baceriophage shows the same value for either the latent period or the burst size on both host cells. This indicates that the difference of the plaque size of the phage MT produced on S. typhi (783) and S. pullorum (Nakamura) can not be explained by the difference in the latent period or the burst size, and another factor has to be looked for to make it clear.

Now, let us consider why and how the plaque size, in general, has a definite value in average in a definite host phage system. If the host cells are at any 


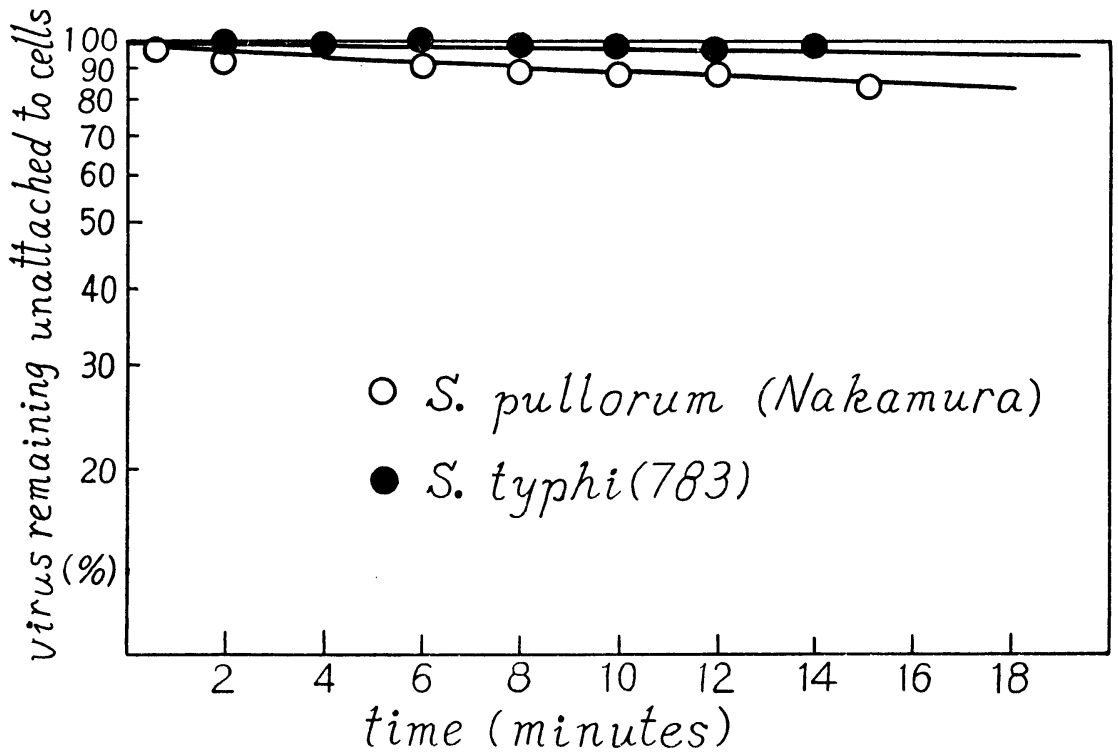

Fig. 2. Adsorption-time curves of the phage MT to S. pullorum (Nakamura) and to S. typhi (783)

time susceptible to the phage, the plaques should continue to increase their size indefinitely, but it is never the case. This should be the indication of the fact that the host cells cease to be susceptible to the phage infection in a certain period of their growth on a nutrient agar plate. It was stated(6) that there was no liberation of phage T5 by bacteria infected and kept in nutrient broth at concentrations of $2 \times 109$ cells per $\mathrm{ml}$ or higher. This fact may be correlated with the above consideration. Generally speaking, the bacterial growth curve is divided into three parts; namely, the lag phase, the logarithmic phase, and the stationary phase. Thus it may be assumed that the host cells growing logarithmically are susceptible to the phage infection and capable of liberating phage particles, while their capacity to liberate phage is reduced and finally stopped with the stationary phase. Considering in this way, it is concluded that the plaque size may depend to a certain extent upon the length of the logarithmic growth phase or the length of the period until a certain point of the stationary phase of the host cells.

From the above consideration, we undertook an experiment for determining the generation time both of $S$. typhi (783) and $S$. pullorum (Nakamura) in ordinary nutrient broth. The result is shown in Fig. 4 as an usual growth curve for each. According to this experiment, the generation time of $S$. typhi (783) is 15 20 minutes in broth, while that of $S$. pullorum (Nakamura) is $25 \sim 30$ 


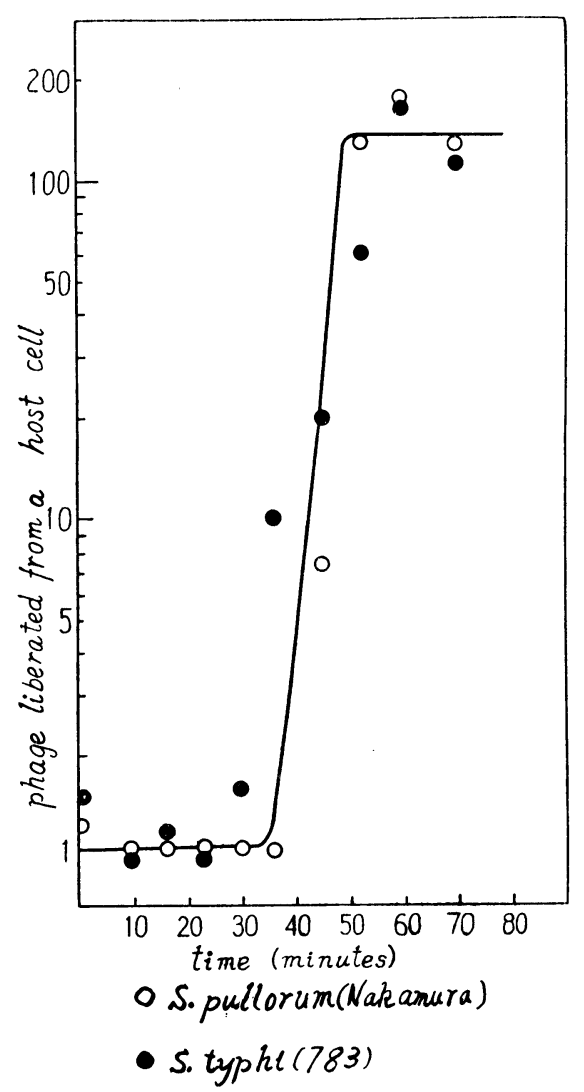

Fig. 3. One-step growth curves of the phage MT on S. pullorum (Nakamura) and S. typhi (783)

minutes in the same medium. As considered above, the difference of the generation time may be the factor influencing the plaque size of the phage MT on both host cells.

\section{Discussion}

The plaque size of a bacteriophage may be influenced by many factors. It is very reasonable to consider that the latent period or the burst size is also one of the important factors for it, and our pervious report concerning the slow-lysis mutant of a host microorganism presented an instance where the latent period was indeed one of the controlling factors for the plaque size.

On the other hand, it is also reasonable that the plaque will continue to grow during the period in which host cells are susceptible to phage infection, and that this period may correlated with their growth phase, and consequently the length of the period of the plaque growth may depend more or less upon the length of the generation time of the host organism. In conclusion, the plaque size may more or less be influenced by the generation period of the host micro- 


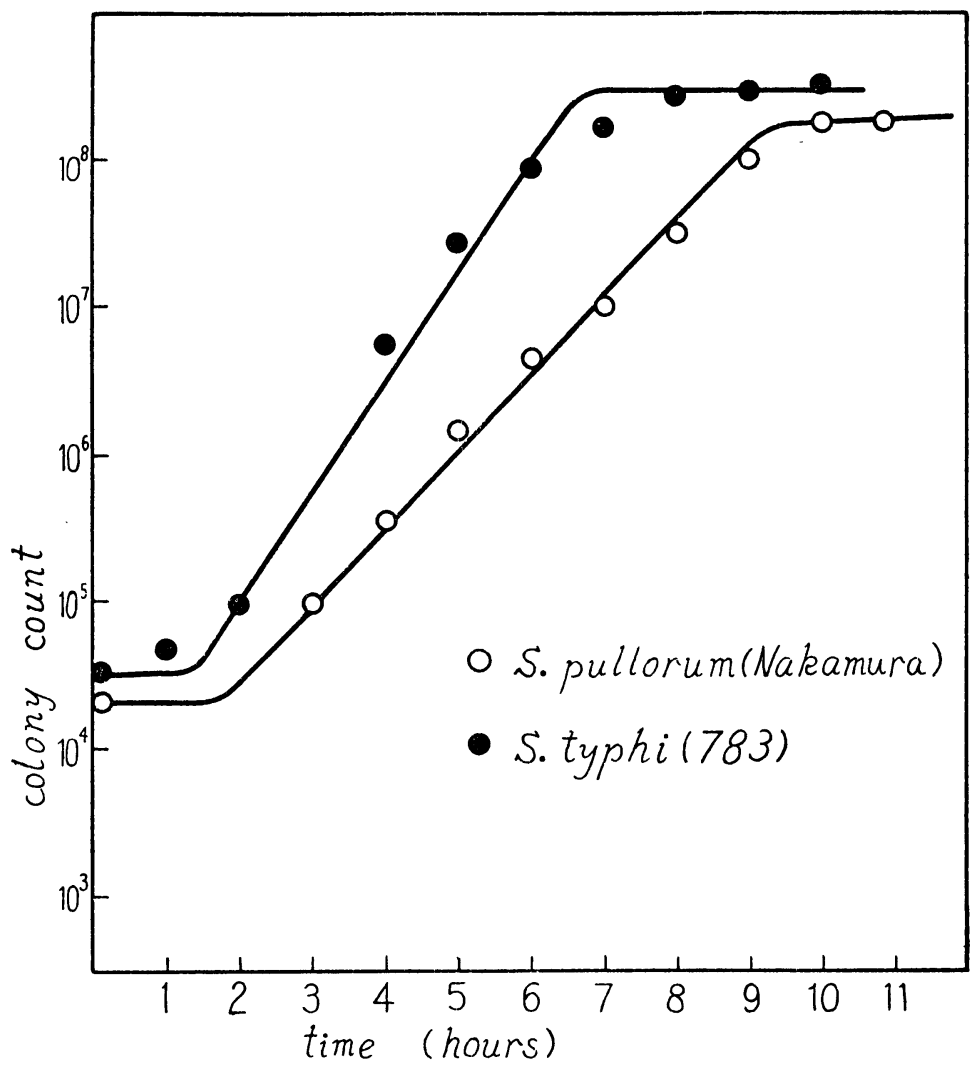

Fig. 4. Growth curves of S. pullorum (Nakamura) and S. typhi (783)

organism. The plaque-size difference of the phage MT on $S$. pullorum and $S$. typhi may be explained as an instance of this consideration.

\section{SUMMARY}

The experiment was carried out on the relationship between the plaque size and the generation time of the host microorganism and the discussion was given

\section{REFERENCES}

1) Nojima, T., and Fukumi, H.: On the variations of the plaque size in the host parasite system of the bacteriophage P 84., Jap. J. Med. Sci. Biol., in press.

2) Fukumi, H., Nojima, T., and Sayama, E.: On the role of the bacteriophage in the change of the antigenic structure in the $\mathrm{E}$ group of Salmonella., Jap. J. Med. Sci. Biol., in press.

3) Adams, M.H.: Methods of study of bacterial viruses, Methods in Medical Research, $2,1-73,1950$.

4) Fukumi, H., and Nojima, T.: An essential feature of host range mutation.,Third report of host-controlled variation-., Jap, J, Med, Sci. Biol., in press.

5) Puck, T.T., Garen, A., and Cline, J.: The mechanism of virus attachment to 
host cells., I. The role of ions in the primary reaction., J. Exp. Med., 93, 65-88, 1951.

6) Luria, S. E., and Steiner, D, L.: The role of calcium in the penetration of bacteriophage $T_{5}$ into its host., J. Bact., 67, 635-639, 1954. 


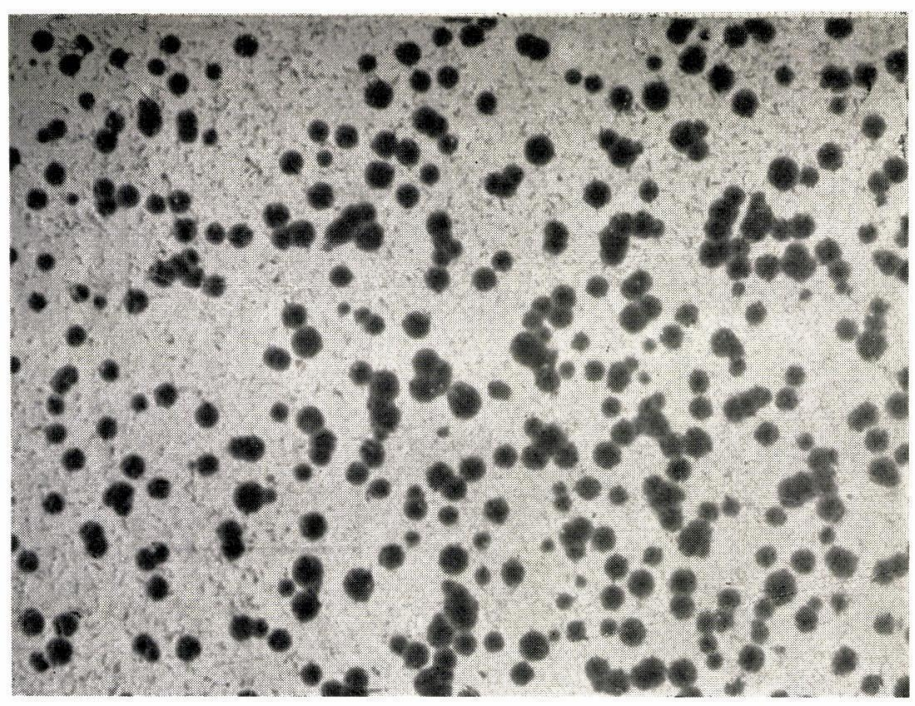

(a) The plaques of the phage MT on S. pullorum (Nakamura)

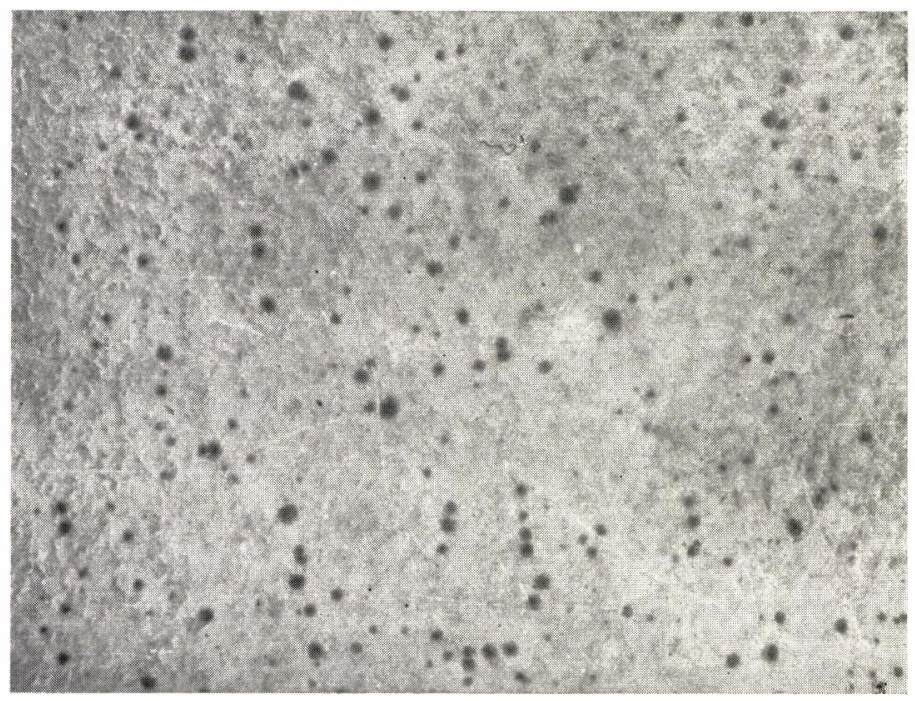

(b) The plaques of the phage MT on S. typhi (783)

Fig. 1. The plaques of the phage MT on $S$. pullorum (Nakamura) and S. typhi (783) 